\title{
BOUNDS FOR EXTREME SINGULAR VALUES OF A COMPLEX MATRIX AND ITS APPLICATIONS
}

\author{
AYŞE DILEK GÜNGÖR AND RAMAZAN TÜRKMEN
}

Abstract. In this study, we have obtained bounds for extreme singular values of a complex matrix $A$ of order $n \times n$.

In addition, we have found a bounds for the extreme singular values of Hilbert matrix, its Hadamard square root, Cauchy-Toeplitz matrix, Cauchy-Hankel matrix in the forms

$$
\begin{aligned}
& H=(1(i+j-1))_{i, j=1}^{n}, \\
& H^{\circ 1 / 2}=\left(1(i+j-1)^{1 / 2}\right)_{i, j=1}^{n}, \\
& T_{n}=[1(g+(i-j) h)]_{i, j=1}^{n} \quad \text { and } \quad H_{n}=[1(g+(i+j) h)]_{i, j=1}^{n},
\end{aligned}
$$

respectively.

Mathematics subject classification (2000): 15A18, 15A45, 15A57, 15A60.

Key words and phrases: Hilbert matrix, Hadamard square root of Hilbert matrix, Toeplitz matrix, Hankel matrix, singular value, lower bound, upper bound.

\section{REFERENCES}

[1] D. BozKuRT, On the $l_{p}$ norms of Cauchy-Toeplitz matrices, Linear and Multilinear, 44, (1998), 341-346.

[2] D. BOZKURT, On the bounds for the $l_{p}$ norm of almost Cauchy-Toeplitz matrix, Turkish Journal of Mathematics, 20, (4) (1996), 545-552.

[3] R. MOENCK, On computing closed forms for summations, Proceedings of the MACSYMA User's Conference ,(1977), 225-236.

[4] S. V. PARTER, On the distribution of the singular values of Toeplitz, matrices, Linear Algebra and its Appl., 80, (1986), 115-130.

[5] E. E. TYRTYSHNIKOV, Cauchy-Toeplitz matrices and some applications, Linear Algebra and its Applications, 149, (1991), 1-18.

[6] R. REAMS, Hadamard inverses, square roots and product of almost semidefinite matrices, Linear Algebra and Its Applications, 288 , (2000), 35-43.

[7] S. SOLAK, R. TURKMEN AND D. BOZKURT, On GCD, LCM and Hilbert matrices and their applications, Applied Mathematics and Computation, (2003).

[8] OSCAR ROJO, Further bounds for the smallest singular value and the spectral condition number, Computers and Mathematics with Applications, 38, 7-8 (1999), 215-228.

[9] A. D. GÜNGÖR, Lower bounds for the norms of Cauchy-Toeplitz and Cauchy-Hankel matrices, Applied Mathematics and Computation (to appear).

[10] S. YONGZHONG, Estimations for the eigenvalues of the product of matrices (Chinese), J. Nanjing Norm. Univ. Nat. Sci. Ed. 16, (2) (1994), 10-13.

[11] R. Mathias, The Spectral Norm of a Nonnegative Matrix, Linear Algebra and its Applications, 131, (1990), 269-284 . 